\title{
Gastrointestinal symptoms and weight loss in cancer patients receiving chemotherapy
}

\author{
Karla Sánchez-Lara*, Emilio Ugalde-Morales, Daniel Motola-Kuba and Dan Green \\ Centro Oncológico Integral, Hospital Médica Sur, Puente de Piedra 150, Colonia Toriello Guerra, Tlalpan, México City, \\ CP 14050, Mexico
}

(Submitted 16 November 2011 - Final revision received 24 April 2012 - Accepted 24 April 2012 - First published online 12 June 2012)

\begin{abstract}
Cancer patients receiving chemotherapy have a high risk of malnutrition secondary to the disease and treatment, and $40-80 \%$ of cancer patients suffer from different degrees of malnutrition, depending on tumour subtype, location, staging and treatment strategy. Malnutrition in cancer patients affects the patient's overall condition, and it increases the number of complications, the adverse effects of chemotherapy and reduces the quality of life. The aim of the present study was to evaluate weight-loss prevalence depending on the tumour site and the gastrointestinal (GI) symptoms of oncology patients receiving chemotherapy. We included 191 cancer patients receiving chemotherapy. Files of all patients were reviewed to identify symptoms that might potentially influence weight loss. The nutritional status of all patients was also determined. The cancer sites in the patients were as follows: breast (31.9\%); non-colorectal GI (18.3\%); colorectal (10.4\%); lung (5.8\%); haematological (13.1\%); others (20.5\%). Of these patients, $58 \%$ experienced some degree of weight loss, and its prevalence was higher among the non-colorectal GI and lung cancer patients. Common symptoms included nausea (59.6\%), anorexia (46\%) and constipation (31.9\%). A higher proportion of patients with $\geq 5 \%$ weight loss experienced anorexia, nausea and vomiting (OR 9.5, $2 \cdot 15$ and $6 \cdot 1$, respectively). In conclusion, these results indicate that GI symptoms can influence weight loss in cancer patients, and they should be included in early nutritional evaluations.
\end{abstract}

Key words: Cancer: Gastrointestinal symptoms: Weight loss

Wasting syndrome is common in cancer, and it affects about $50 \%$ of all patients at the time of diagnosis ${ }^{(1,2)}$ and $>80 \%$ of patients in advanced stages ${ }^{(3,4)}$. Weight loss and malnutrition in cancer patients increase the risk of infection and the cost of health care ${ }^{(5,6)}$, decrease the quality of $l i f e^{(7)}$, and affect the response to anti-neoplastic treatment and overall survival $^{(8)}$. The early detection of malnutrition in cancer patients is important, and could increase the overall survival and quality of life ${ }^{(9)}$. Nutritional screening includes anthropometric parameters (BMI and weight loss percentage) and biochemical parameters. However, it is not feasible to interpret some parameters ${ }^{(10)}$ because they are influenced by other non-nutritional factors such as hydration status, tumour site and liver disease. Albumin may not be a good indicator of nutritional state because it is influenced by stress and illness ${ }^{(11)}$. Low serum albumin levels can also occur in patients receiving chemotherapy due to the effects of cytotoxic agents ${ }^{(12)}$.

Routine screening of malnutrition in cancer patients should be non-invasive and should include factors such as gastrointestinal (GI) symptoms that affect food intake and eating behaviour
The aim of the present study was to evaluate weight-loss prevalence in cancer patients receiving chemotherapy based on the tumour site and the importance of GI symptoms in the presence of malnutrition.

\section{Methods}

The present retrospective study was conducted at the Medica Sur University Hospital's Oncology Centre, between January 2008 and July 2010. We reviewed the files of all patients treated with chemotherapy at this centre. Biochemical and clinical data were assessed after the second chemotherapy cycle.

The selection criteria were as follows: age between 19 and 75 years; complete diagnosis information (primary cancer site, type of tumour and stage at diagnosis); complete information regarding the main GI symptoms that occurred during chemotherapy, which were reported by the patient's clinical oncology doctor (e.g. nausea, vomiting, diarrhoea, constipation and anorexia); complete information for anthropometric variables (weight, height, habitual weight, and weight loss during the past 6 months). The exclusion criteria included

Abbreviation: GI, gastrointestinal.

*Corresponding author: K. Sánchez-Lara, email ksanchez@medicasur.org.mx 
Table 1. General characteristics

(Mean values and standard deviations; number of patients and percentage values)

\begin{tabular}{lccc}
\hline Variables & $n$ & & $\%$ \\
\hline Sex & & & \\
$\quad$ Male & 68 & & $35 \cdot 6$ \\
$\quad$ Female & 123 & & $64 \cdot 4$ \\
Age (years) & & & \\
$\quad$ Mean & & $54 \cdot 3$ & \\
SD & & $14 \cdot 8$ & \\
Weight (kg) & & \\
$\quad$ Mean & & $68 \cdot 2$ & \\
SD & & 14.6 & \\
BMl (kg/m $\left.{ }^{2}\right)$ & & \\
$\quad$ Mean & & 25.4 & \\
SD & & 4.9 & \\
Weight loss (kg) & & 4.5 & \\
$\quad$ Mean & & 4.5 & \\
SD & & & \\
Weight loss & & \\
$\quad \geq 5 \%$ & 74 & & \\
$\geq 10 \%$ & 47 & & \\
& & & \\
\end{tabular}

incomplete files, patients who received radiotherapy treatment or a history of surgery as an oncological treatment. For descriptive purposes, patients were classified by their tumour sites as follows: colon; lung; breast; non-colorectal GI (gastric, oesophageal, gall bladder and pancreatic); gynaecological (cervico-uterine and ovarian); haematological (lymphoma, leukaemia and myeloma); others (prostate, thyroid and testicular). The present study was conducted according to the guidelines laid down in the Declaration of Helsinki and all procedures involving human subjects/patients were approved by the Medica Sur ethics committee.

\section{Statistical analysis}

Descriptive statistics were used for qualitative and quantitative variables: frequency; percentage; mean and standard deviation. $\chi^{2}$ and Mann-Whitney $U$ tests were applied to parametric and non-parametric data, respectively. Data analysis was performed using the SPSS program version 18 (SPSS, Inc.). $P \leq 0.05$ was considered significant.

\section{Results}

We reviewed 534 files of all patients who had undergone chemotherapy, of which a total of 191 files met the inclusion criteria, including 123 females and sixty-eight males. The most frequent primary tumour site was breast cancer (31.9\%). The mean age was 54.3 (SD 14.8) (range 28-72) years. The mean body weight was 68.0 (SD 14.6) $\mathrm{kg}$ and the mean BMI was $25.4(\mathrm{sD} 4.9) \mathrm{kg} / \mathrm{m}^{2}$. Over $63 \%$ of the patients experienced unintentional weight loss, while 38.7 and $24.6 \%$ of the patients had $\geq 5 \%$ and $\geq 10 \%$ weight loss, respectively, during the 6 months before the treatment (Table 1). Weight loss during the 6 months before the treatment had a higher prevalence in patients with gynaecological and GI cancers (91.6 and $77.1 \%$, respectively; Table 2). Patients with noncolorectal GI cancer had higher rates of weight loss after two cycles of chemotherapy $(\geq 10 \%$ of habitual weight; Table 2). The most common GI symptoms reported in all study patients were nausea (59.6\%) and anorexia (46\%). Patients with $\geq 5 \%$ weight loss were significantly associated with nausea (OR 2.15, $P=0 \cdot 03$ ), vomiting (OR 6.1, $P=0 \cdot 017$ ) and anorexia (OR 9.5, $P=0 \cdot 003$ ). Patients with $\geq 10 \%$ weight loss were significantly associated with vomiting (OR $4 \cdot 3$, $P=0 \cdot 05$ ) and anorexia (OR 13.4, P<0.001) (Table 3).

\section{Discussion}

The prevalence of unintentional weight loss in patients with cancer has been reported as $49-74 \%$, and is associated with the primary tumour location ${ }^{(13-15)}$. The Eastern Cooperative Oncology Group ${ }^{(16)}$ and others studies ${ }^{(17,18)}$ have reported that patients with gastric cancer have the highest rates of weight loss, whereas patients with breast cancer have the lowest rates. The present study found similar results, and more than $50 \%$ of our patients experienced weight loss. The highest prevalence was found with GI (77\%), lung (72.7\%) and gynaecological tumours (91.6\%). The lowest prevalence was observed in colon and breast cancer patients. The mean BMI of Mexican patients with a cancer diagnosis has been reported as $25.5 \mathrm{~kg} / \mathrm{m}^{2(19)}$. In the present study, the mean BMI of the patients was $>25 \mathrm{~kg} / \mathrm{m}^{2}$, with the exception of non-colorectal GI and gynaecological cancer patients. Therefore, the majority of patients would have been classified as

Table 2. Weight, BMI and weight loss by tumour location

(Mean values and standard deviations; number of patients and percentages, $n$ 191)

\begin{tabular}{|c|c|c|c|c|c|c|c|c|c|c|c|}
\hline \multirow[b]{2}{*}{ Type of cancer } & \multirow[b]{2}{*}{$n$} & \multirow[b]{2}{*}{$\%$} & \multicolumn{2}{|c|}{ Weight (kg) } & \multicolumn{2}{|c|}{ BMl $\left(\mathrm{kg} / \mathrm{m}^{2}\right)$} & \multicolumn{5}{|c|}{ Patients with weight loss } \\
\hline & & & Mean & SD & Mean & $\mathrm{SD}$ & $<5 \%(n 44)$ & $5-10 \%(n 26)$ & $\geq 10 \%(n 46)$ & Total & $\%$ \\
\hline $\mathrm{Gl}$ & 35 & $18 \cdot 3$ & 64.9 & $14 \cdot 0$ & 23.6 & $4 \cdot 7$ & 3 & 5 & 19 & 27 & $77 \cdot 1$ \\
\hline Colon & 20 & $10 \cdot 4$ & 69.9 & $17 \cdot 7$ & $25 \cdot 7$ & 4.0 & 3 & 5 & 1 & 9 & $45 \cdot 0$ \\
\hline Lung & 11 & $5 \cdot 8$ & $69 \cdot 3$ & $17 \cdot 8$ & $25 \cdot 3$ & $6 \cdot 0$ & 3 & 2 & 3 & 8 & $72 \cdot 7$ \\
\hline Breast & 61 & $31 \cdot 9$ & $67 \cdot 1$ & $12 \cdot 7$ & $26 \cdot 1$ & $4 \cdot 8$ & 20 & 3 & 4 & 27 & 47.5 \\
\hline Gynecological & 12 & $6 \cdot 3$ & $57 \cdot 8$ & 10.5 & $22 \cdot 9$ & 3.3 & 3 & 4 & 4 & 11 & 91.6 \\
\hline Others & 27 & $14 \cdot 1$ & $74 \cdot 1$ & 14.5 & $26 \cdot 6$ & 4.5 & 11 & 4 & 5 & 20 & 74.1 \\
\hline Haematological & 25 & $13 \cdot 1$ & 73.0 & $13 \cdot 7$ & $25 \cdot 7$ & 4.5 & 1 & 3 & 6 & 10 & $40 \cdot 0$ \\
\hline Total & 191 & 100 & $68 \cdot 0$ & $14 \cdot 7$ & $25 \cdot 1$ & 4.9 & 42 & 26 & 42 & 112 & 58.6 \\
\hline
\end{tabular}

Gl, gastrointestinal. 
Table 3. Association between unintentional weight loss and gastrointestinal (GI) symptoms

(Odds ratios and $P$ values)

\begin{tabular}{|c|c|c|c|c|}
\hline \multirow[b]{3}{*}{ Gl symptoms } & \multicolumn{4}{|c|}{ Weight loss } \\
\hline & \multicolumn{2}{|c|}{$\geq 5 \%$} & \multicolumn{2}{|c|}{$\geq 10 \%$} \\
\hline & OR & $P$ & OR & $P$ \\
\hline Nausea & $2 \cdot 15$ & 0.032 & 3.4 & 0.06 \\
\hline Vomiting & $6 \cdot 1$ & 0.017 & $4 \cdot 3$ & 0.05 \\
\hline Diarrhoea & $1 \cdot 15$ & 0.871 & 1.28 & 0.651 \\
\hline Constipation & 1.45 & 0.516 & $1 \cdot 21$ & 0.276 \\
\hline Anorexia & 9.5 & 0.003 & 13.4 & $<0.001$ \\
\hline
\end{tabular}

overweight or obese without any malnutrition risk if we considered only the BMI as a nutritional parameter; however, the BMI classification ignores body composition. Recent research into the body composition of cancer patients, specifically the proportion of lean and fat tissue, has demonstrated that cancer patients may experience sarcopenic obesity (simultaneous obesity and low muscle mass) ${ }^{(20)}$. Therefore, it is essential to consider other tools to obtain a good nutritional evaluation, such as a recent history of weight change (i.e. proportional weight loss during the last 6 months, 2 weeks or compared with the habitual weight). Unintentional weight loss is a more powerful parameter than BMI for malnutrition detection in cancer patients ${ }^{(21,22)}$. Subjective Global Assessment and body composition measurements using bioelectrical impedance or computerised tomography are very useful $^{(20,23,24)}$. The present study considered the percentage of weight loss, and most patients experienced a $5 \%$ reduction in their habitual weight. Based on these results, we conclude that weight loss may be a better malnutrition parameter than BMI in patients with cancer who are receiving chemotherapy. Marin et al. ${ }^{(25)}$ evaluated 226 oncology patients, and 10 and $64 \%$ of patients presented malnutrition based on a consideration of BMI and Subjective Global Assessment, respectively. Similar studies have reported a malnutrition prevalence of $6-15 \%$ based on $\mathrm{BMI}^{(26)}$, whereas the prevalence was higher when Subjective Global Assessment was applied ${ }^{(27-29)}$. These variations could be explained by changes in water distribution due to tumours (oedema, ascites, dehydration, diarrhoea and vomiting), or accelerated tumour growth ${ }^{(30)}$. Other mechanical and metabolic complications can occur in addition to the primary tumour site. GI symptoms are important components of malnutrition in cancer patients. In the present study, the most frequent GI symptoms were nausea (59.6\%), anorexia (46\%) and constipation (31.9\%). A similar prevalence of GI symptoms was found in studies conducted by Tchekmedyian et al. ${ }^{(14)}$, Segura et $a l .{ }^{(15)}$, Walsh et $a l .{ }^{(31)}$ and others ${ }^{(32-34)}$. In the present study, nausea, anorexia and vomiting were significantly associated with $\geq 5 \%$ weight loss, whereas diarrhoea and constipation were not associated. These symptoms could result in weight loss by changing the body water distribution. The use of a retrospective series and the under-reporting of toxicity symptoms were limitations of the present study. Minor GI symptoms such as dysphagia, taste disorders and early satiety were reported inconsistently in the patients' files. The poor reporting of toxicity or its symptoms is common following cancer treatments. The association of GI symptoms with nutritional status could be relevant, and should be considered in further studies. It was also important that only about one-third of patients could be included because missing basic clinical data, such as weight, were not measured or recorded. This data deficiency must be addressed in clinical practice because of the implications of malnutrition for cancer patients' outcomes.

\section{Conclusion}

In our population, non-colorectal GI and lung cancer patients had a higher prevalence of weight loss compared with patients with other primary cancer sites. GI symptoms such as anorexia, nausea and vomiting were significantly correlated with weight loss. The present study confirmed that the primary tumour site, weight-loss history and GI symptoms were determinant factors of nutritional status in oncology patients, and they must be included in the screening, evaluation and treatment of cancer patients.

\section{Acknowledgements}

This study received no financial support. The authors declare that there are no conflicts of interest. The authors' contributions are as follows: U.-M. E. and M.-K. D. participated in the data collection; S.-L. K. was involved in writing and analysis; G. D. contributed to the writing and editing assistance.

\section{References}

1. Gomez Candela C, Olivar Roldan J, Garcia M, et al. (2010) Assessment of a malnutrition screening tool in cancer patients. Nutr Hosp 25, 400-405.

2. Gomez-Candela C, Luengo LM, Cos AI, et al. (2003) Subjective global assessment in neoplastic patients. Nutr Hosp 18, 353-357.

3. Agius R, Nadulski T, Kahl HG, et al. (2010) Validation of a headspace solid-phase microextraction-GC-MS/MS for the determination of ethyl glucuronide in hair according to forensic guidelines. Forensic Sci Int 196, 3-9.

4. Trabal J, Leyes P, Forga MT, et al. (2006) Quality of life, dietary intake and nutritional status assessment in hospital admitted cancer patients. Nutr Hosp 21, 505-510.

5. Bossola M, Pacelli F, Tortorelli A, et al. (2007) Cancer cachexia: it's time for more clinical trials. Ann Surg Oncol 14, 276-285.

6. Murry DJ, Riva L \& Poplack DG (1998) Impact of nutrition on pharmacokinetics of anti-neoplastic agents. Int $J$ Cancer Suppl 11, 48-51.

7. Laky B, Janda M, Kondalsamy-Chennakesavan S, et al. (2010) Pretreatment malnutrition and quality of life - association with prolonged length of hospital stay among patients with gynecological cancer: a cohort study. BMC Cancer 10, 232-237.

8. Capra S, Ferguson M \& Ried K (2001) Cancer: impact of nutrition intervention outcome - nutrition issues for patients. Nutrition 17, 769-772. 
9. Sosa-Sanchez R, Sanchez-Lara K, Motola-Kuba D, et al. (2008) The cachexia-anorexia syndrome among oncological patients. Gac Med Mex 144, 435-440.

10. Gupta D, Lammersfeld CA, Vashi PG, et al. (2005) Prognostic significance of Subjective Global Assessment (SGA) in advanced colorectal cancer. Eur J Clin Nutr 59, 35-40.

11. Wong PW, Enriquez A \& Barrera R (2001) Nutritional support in critically ill patients with cancer. Crit Care Clin 17, 743-767.

12. Arrieta O, Michel Ortega RM, Villanueva-Rodriguez G, et al. (2010) Association of nutritional status and serum albumin levels with development of toxicity in patients with advanced non-small cell lung cancer treated with paclitaxel-cisplatin chemotherapy: a prospective study. $B M C$ Cancer 10, 50

13. Dufau L (2010) Prevalencia de desnutrición en pacientes oncológicos (Prevalence of malnutrition in cancer patients). Diaeta 28, 31-36

14. Tchekmedyian NS (1995) Costs and benefits of nutrition support in cancer. Oncology 9, 79-84.

15. Segura A, Pardo J, Jara C, et al. (2005) An epidemiological evaluation of the prevalence of malnutrition in Spanish patients with locally advanced or metastatic cancer. Clin Nutr 24, 801-814

16. Dewys WD, Begg C, Lavin PT, et al. (1980) Prognostic effect of weight loss prior to chemotherapy in cancer patients. Eastern Cooperative Oncology Group. Am J Med 69, 491-497.

17. Zorlini R, Akemi Abe Cairo A \& Salete Costa Gurgel M (2008) Nutritional status of patients with gynecologic and breast cancer. Nutr Hosp 23, 577-583.

18. Laky B, Janda M, Bauer J, et al. (2007) Malnutrition among gynaecological cancer patients. Eur J Clin Nutr 61, 642-646.

19. Fuchs V \& Gutierrez G (2008) Situación nutricional en pacientes oncológicos internados en un hospital público de la ciudad de México (Nutritional status in cancer patients admitted to a public hospital in Mexico City). Rev Cubana Med 47, 1-5.

20. Prado CM, Lieffers JR, McCargar LJ, et al. (2008) Prevalence and clinical implications of sarcopenic obesity in patients with solid tumours of the respiratory and gastrointestinal tracts: a population-based study. Lancet Oncol 9, 629-635.

21. Lipkin EW \& Bell S (1993) Assessment of nutritional status. The clinician's perspective. Clin Lab Med 13, 329-352.

22. Gudny Geirsdottir O \& Thorsdottir I (2008) Nutritional status of cancer patients in chemotherapy; dietary intake, nitrogen balance and screening. Food Nutr Res 52 (Epublication ahead of print version 12 December 2008).

23. Prado CM, Baracos VE, McCargar LJ, et al. (2009) Sarcopenia as a determinant of chemotherapy toxicity and time to tumor progression in metastatic breast cancer patients receiving capecitabine treatment. Clin Cancer Res 15, 2920-2926.

24. Prado CM, Baracos VE, McCargar LJ, et al. (2007) Body composition as an independent determinant of 5-fluorouracilbased chemotherapy toxicity. Clin Cancer Res 13, 3264-3268.

25. Marin Caro MM, Gomez Candela C, Castillo Rabaneda R, et al. (2008) Nutritional risk evaluation and establishment of nutritional support in oncology patients according to the protocol of the Spanish Nutrition and Cancer Group. Nutr Hosp 23, 458-468.

26. Collins MM, Wight RG \& Partridge G (1999) Nutritional consequences of radiotherapy in early laryngeal carcinoma. Ann $R$ Coll Surg Engl 81, 376-831.

27. Laky B, Janda M, Cleghorn G, et al. (2008) Comparison of different nutritional assessments and body-composition measurements in detecting malnutrition among gynecologic cancer patients. Am J Clin Nutr 87, 1678-1685.

28. Jagoe RT, Goodship TH \& Gibson GJ (2001) The influence of nutritional status on complications after operations for lung cancer. Ann Thorac Surg 71, 936-943.

29. Edington J, Winter PD, Coles SJ, et al. (1999) Outcomes of undernutrition in patients in the community with cancer or cardiovascular disease. Proc Nutr Soc 58, 655-661.

30. Sánchez-Lara K, Turcott J, Sosa-Sánchez R, et al. (2008) Evaluación del estado de nutrición en el paciente con cancer (Assessment of nutritional status in patients with cancer). Rev Endocrinol Nut 16, 165-171.

31. Walsh D, Donnelly S \& Rybicki L (2000) The symptoms of advanced cancer: relationship to age, gender, and performance status in 1,000 patients. Support Care Cancer $\mathbf{8}$ $175-179$

32. Grosvenor M, Bulcavage L \& Chlebowski RT (1989) Symptoms potentially influencing weight loss in a cancer population. Correlations with primary site, nutritional status, and chemotherapy administration. Cancer 63, 330-334.

33. Cid Conde L, Fernandez Lopez T, Neira Blanco P, et al. (2008) Hyponutrition prevalence among patients with digestive neoplasm before surgery. Nutr Hosp 23, 46-53.

34. Sarhill N, Mahmoud F, Walsh D, et al. (2003) Evaluation of nutritional status in advanced metastatic cancer. Support Care Cancer 11, 652-659. 